\title{
Robotic rehabilitation for end-effector device and botulinum toxin in upper limb rehabilitation in chronic post-stroke patients: an integrated rehabilitative approach
}

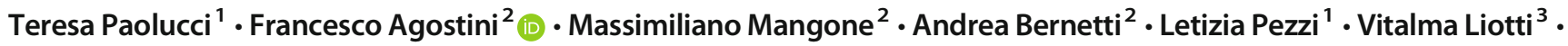 \\ Elena Recubini ${ }^{3} \cdot$ Cristina Cantarella $^{3} \cdot$ Rosa Grazia Bellomo ${ }^{4} \cdot$ Carlo D'Aurizio $^{3} \cdot$ Raoul Saggini $^{1,5}$
}

Received: 24 September 2020 / Accepted: 13 March 2021 / Published online: 7 April 2021

(C) The Author(s) 2021

\begin{abstract}
Background Determine the effects of an integrated rehabilitation protocol, including botulinum toxin and conventional rehabilitation exercise plus end-effector (EE) robotic training for functional recovery of the upper limb (UL) compared to training with the robot alone in post-chronic stroke patients with mild to severe spasticity, compared to training with the robot alone.

Methods In this prospective, observational case-control study, stroke patients were allocated into 2 groups: robot group (RG, patients who underwent robotic treatment with EE) and robot-toxin group (RTG, patients who in addition have carried out the injection of botulinum toxin for UL recovery). All patients were assessed by Fugl-Meyer Assessment (FMA), Motricity Index (MI), modified Ashworth scale (MAS), numeric rating scale (NRS), Box and Block Test (BBT), Frenchay Arm Test (FAT), and Barthel Index (BI) at baseline (T0), T1 (end of treatment), and T2 (3 months of follow-up).

Results Forty-four patients were included and analyzed (21RG; 23RTG). From the analysis between groups, the results suggested how there was a statistically significant difference in favor of RTG, specifically $\Delta \mathrm{T} 0$-T1 and $\Delta \mathrm{T} 0$-T2 for B\&B $p=0.009$ and $p=0.035 ; \Delta \mathrm{T} 0$-T1 and $\Delta \mathrm{T} 0$-T2 for FAT with $p=0.016$ and $p=0.031 ; \Delta \mathrm{T} 0$-T1 for MAS shoulder $p=0.016 ; \Delta \mathrm{T} 0$-T1 and $\Delta \mathrm{T} 0$-T2 with $p=0.010$ and $p=0.005$ for MAS elbow; and $\Delta \mathrm{T} 0$-T1 and $\Delta \mathrm{T} 0$-T2 with $p=0.001$ and $p=0.013$ for MAS wrist. Conclusion Our results suggest, in line with the literature, a good efficacy in the reduction of spasticity and in the improvement of the function of the UL, with the reduction of pain, adopting a rehabilitation protocol integrated with BoTN, robot-assisted training, and traditional physiotherapy.
\end{abstract}

Keywords Evaluation model $\cdot$ Motion control skill $\cdot$ Robotic end-effector kinematic feature $\cdot$ Surgical robot system

Massimiliano Mangone

massimiliano.mangone@uniroma1.it

Francesco Agostini

francescoagostini.ff@gmail.com

1 Unit of Physical Medicine and Rehabilitation, Department of Oral Medical Science and Biotechnology (DSMOB), G. D’Annunzio University of Chieti-Pescara, Chieti, Italy

2 Physical Medicine and Rehabilitation Unit, Department of Anatomical and Histological Sciences, Legal Medicine and Orthopedics, Sapienza University of Rome, Piazzale Aldo Moro 5, 00185 Rome, Italy

3 U.O.C. Physical Medicine and Rehabilitation, Hospital of Popoli, Pescara, Italy

4 Department of Biomolecular Sciences, University of Study of Urbino Carlo Bo, Urbino, Italy

5 IRCSS Centro Neurolesi "Bonino Pulejo”, Messina, Italy

\section{Introduction}

Rehabilitation represents a very important focus for motor recovery after stroke which influences the neurobiology of neuronal plasticity providing controlled, repetitive, and variable patterns. After a stroke, approximately $80 \%$ of patients report a UL motor deficit with all subsequent limitations in activities of daily living and limiting social participation $[1,2]$.

For the functional recovery of the upper limb (UL), training with specific repetitive task exercises with progressive difficulty, goal oriented, is recommended. In addition, taskoriented exercises in the recovery of UL function should be tailored and personalized as much as possible considering the patient's possibilities and needs $[3,4]$. From these premises, robotic devices for UL open a window to define therapeutic modalities as a possible beneficial drug, in patients with a moderate-to-severe deficit after stroke, able to boost 
biological, neurobiological, and epigenetic changes in the central nervous system (CNS) [5]. We can consider three main functional consequences of impairments on UL function after stroke: (1) learned nonuse, (2) learned bad use, and (3) forgetting use [6], where robotic rehabilitation with both the endeffector system (EE) and the exoskeleton (ES) can be effective in the recovery alone or in association with conventional therapy. For example, during rhythmic pointing movements of the UL and the hand, end-effector and joint angles are reciprocally related in synergies and the action system is organized as a complex dynamical system [7-9]; then, robotic training exercises could improve the fine and purposeful motor organization of the UL as in acute as in chronic phase after stroke. Moreover, recovery of the UL function recognizes a recovery time of over 1 year that is likely mediated by a complex combination of spontaneous and learning-dependent processes, including restitution, substitution, and compensation. In particular, upper extremity impairments have chronic effects on functional independence and satisfaction in 50 to $70 \%$ of all stroke patients $[10,11]$. Therefore, the rehabilitation of $U L$ in the post-stroke patient represents a very important aspect to integrate with conventional therapy. From this point of view, robotic therapy represents a rehabilitation resource that has given very encouraging results in recent years. The UL robotic-assisted therapy combined with conventional therapy during the early rehabilitation phase after stroke is more effective than conventional therapy alone to improve gross manual dexterity, upper limb ability during functional tasks, and patient social participation $[12,13]$. Also, Stephanie Hyeyoung Lee and colleagues studied that the EE robot intervention is better than the Exo-robot intervention with regard to activity and participation among chronic stroke patients with moderate-to-severe UL impairment [14]. Very interesting is the study by Mazzoleni et al., which pointed out how a haptic device in chronic stroke patients reported significant changes on the elbow spasticity [15]. Also, other authors demonstrated improvement in motor function and in muscular activation pattern after a short robotic training in chronic post-stroke spasticity of UL treated prior to the treatment with botulinum toxin; instead, in less severe spasticity, the only robotic treatment could be effective [16]. Deepening the topic, Gandolfi et al. showed that the combined use of robot-assisted UL training and botulinum toxin (BoNT) appears to be a promising therapeutic synergism to improve UL function in chronic stroke patients, but only the robot-assisted UL training contributed to improving muscle strength [17]. The robotic rehabilitation should have to include task-specific and contextspecific training as principles in motor learning, and the training should target the goals that are relevant for the needs of patients.

In the context of pharmacological treatment, in association with the rehabilitation plan, the use of botulinum toxin (BT) is indicated for the treatment of focal spasticity in the hemiplegic patient, especially for the recovery of the UL [18]: BT type A improved muscle tone, physician global assessment, and disability assessment scale in upper limb spasticity and increases the Fugl-Meyer score in lower limb spasticity [19]. Also, Andringa et al. in a recent systematic review concluded that no further trials are needed to investigate BoNT for its favorable effects on resistance to passive movement of the spastic wrist and fingers, and on selfcare [20]. In light of these premises and the indications of the literature regarding UL robotic rehabilitation in the post-stroke patient, the aim of our research was to determine the effects of an integrated rehabilitation protocol, including botulinum toxin and conventional rehabilitation exercise with EE robotic training for functional recovery of UL in post-chronic stroke patients with mild to severe spasticity, compared to training with the robot alone. The primary outcome was the assessment of spasticity and the secondary outcome was the recovery of UL function.

\section{Materials and methods}

Patients with chronic stroke ( $>6$ months) (time from stroke 12-24 months) of both sexes who were attending the outpatient rehabilitative unit of SS Trinità Hospital of Popoli (Italy) from September 2018 to December 2019 were enrolled [21].

The inclusion criteria were previous stroke (ischemic/hemorrhagic) documented at neuroradiological examinations (CT/ MRI) with upper limb functional deficits (associated or not with leg paresis), with a score $>2$ at MRC test, with a sensorymotor deficit, monoparesis, hemiparesis; age > 18 years; Mini-Mental Examination Score > 24 [22-24], BMI < 30; possibility of maintaining the sitting position. Patients were excluded if they presented with any of the following: bilateral impairment; neglect according to barrage test and clinical judgment; cognitive or behavioral impairment such as to affect the understanding or execution of robotic training; inability or unwillingness to give informed consent; severe comorbidities.

The research was approved by the Local Hospital Committee of Popoli and was approved by the Department of Oral and Biotechnological Medical Sciences of the G. D'Annunzio University of Chieti (Italy) (315/20).

The study complies with the principles of the Helsinki Declaration in accordance with good clinical practice. All patients gave written informed consent after receiving detailed information on the study's aims and procedures. Patient personal data are processed anonymously with an alphanumeric code and electronically stored.

Clinical data were collected at T0 (baseline), $\mathrm{T} 1$ (at the end of the treatment), and T2 (after 3 months of follow-up). Adverse events were also registered during the follow-up. 


\section{Study design}

The prospective, observational case-control study was conducted following the Strobe Guidelines [25].

Stroke patients were consecutively enrolled and allocated into 2 groups by 1:1 sample randomization: robot group (RG-patients who underwent robotic treatment with endeffector and conventional treatment) and robot-toxin group (RTG-patients who in addition to robotic treatment and conventional treatment) have carried out the injection of botulinum toxin for upper limb recovery). To reduce bias, the physician who administered the rating scales was blinded to the patient's group allocation, and the researcher who analyzed the data was blinded to the therapy.

All patients in both groups underwent conventional rehabilitation treatment for UL.

\section{BoTN treatment-target muscles and drug dosage}

RTG patients underwent BoNT-A injection in the paretic upper limb. The inoculations were always carried out after clinical and functional evaluation, in order to plan the muscles to be infiltrated and the dose for each muscle also in relation to the patient's response to previous inoculations. In fact, the same administration scheme was not always followed; an example is given by the flexor of the first finger, whose reinoculation was often not necessary. The infiltrations were always performed, after palpation of the muscle bellies, with ultrasound guidance (MYlabfive, ESAOTE) in order to minimize the risk of errors and avoid inoculation into the vessels. The drug dilution was done with $2 \mathrm{CC}$ of saline, and 2 points were infiltrated for each muscle.

We chose to treat the following muscles as necessary: brachial biceps (BB), brachioradialis (BR), pronator teres (PT), flexor carpi radialis (FCR), flexor carpi ulnaris (FCU), flexor digitorum superficialis (FDS), flexor digitorum profundus (FDP), and flexor digitorum longus (FDL). Two commercial formulations of BoNT were used according to hospital availability (Botox ${ }^{\circledR}$-Allergan Inc; Dysport ${ }^{\circledR}$-Ipsen Biopharm Ltd), and the dose injected for each muscle was based on the severity of spasticity, up to the maximum dose approved for the treatment of spasticity of the upper limb in adults. The number of injection sites per muscle and the dose injected into each muscle were determined at the discretion of the investigator, physician in physical medicine, and rehabilitation, according to the USPRM spasticity approach [26] (Table 1).

\section{Rehabilitative treatment}

\section{Robotic end effector}

All outpatients recruited underwent robotic treatment with "MOTORE - MObile roboT for upper limb neuroOrtho
Table 1 Muscles treated and dosages

\begin{tabular}{lll}
\hline Muscle & Dysport (IU) & Botox (IU) \\
\hline Biceps brachial head medial & 100 & 40 \\
Biceps brachialis head lateral & 100 & 40 \\
Subscapularis (in a few cases) & 200 & - \\
Brachialis & $150-200$ & $60-70$ \\
Brachioradialis & $150-200$ & $60-70$ \\
Round pronator & $200-250$ & $70-80$ \\
Radial flexor of the carpus & $150-200$ & $60-70$ \\
Ulnar flexor of the carpus & 150 & 60 \\
1st finger flexor & 150 & 60 \\
Superficial flexor of the fingers & $150-200$ & $60-70$ \\
Deep flexor of the fingers & $150-200$ & $60-70$ \\
\hline
\end{tabular}

$I U$, international unit

REhabilitation" (Humanware, Italy), which is a device for upper limb rehabilitation, offering different modes of operation, ranging from guided support for highly functional patients to assisted or unassisted movement for patients with severe hemiplegia that supports, helps, and/or opposes the movement according to the rehabilitation goals. The device allows patients to perform exercises with visual feedback that let the execution of planar (bidimensional) movements, like reaching and carrying exercises with the intention to rehabilitate the shoulder and elbow areas. The graphical interface allows exercises for carrying out predetermined trajectories (circular or oval) to improve ROM and relearn how to dose strength, exercises that simulate daily life activities (washing dishes, taking coins) and exercises that train cognitive functions (memory, logical deductive functions) (Fig. 1). Considering also the protocol of Aprile et al. [27, 28], the patients performed every evaluation task three times and repeated every reaching movements from 8 to 12 repetitions as follows: (A) trajectories (the patient is asked to drive one of the selectable tracks with his car); (B) pursuit (the patient is asked to chase the opposing car with his car along one of the selectable tracks); (C) coins (the patient is asked to grab some coins and bring them back towards the center of the work surface, where they will accumulate. For this exercise, the patient will perform "fan" movements); (D) memory (the patient is asked to make associations between pairs of images being free to move on the plane without having to follow a particular trajectory); (E) washing dishes (the patient is asked to wash the dishes according to a predetermined sequence of actions); (F) moles (the patient is asked to crush the moles that come out of the ground; $(\mathrm{G})$ archery (the patient is asked to exert an appropriate force on the handpiece of the manipulandum in the opposite direction to that joining the arch and the center of the target, as when loading the bow before firing the arrow) (Fig. 2). The treatment was performed daily for $30 \mathrm{~min}, 3$ days a week, for a total of 20 sessions. 


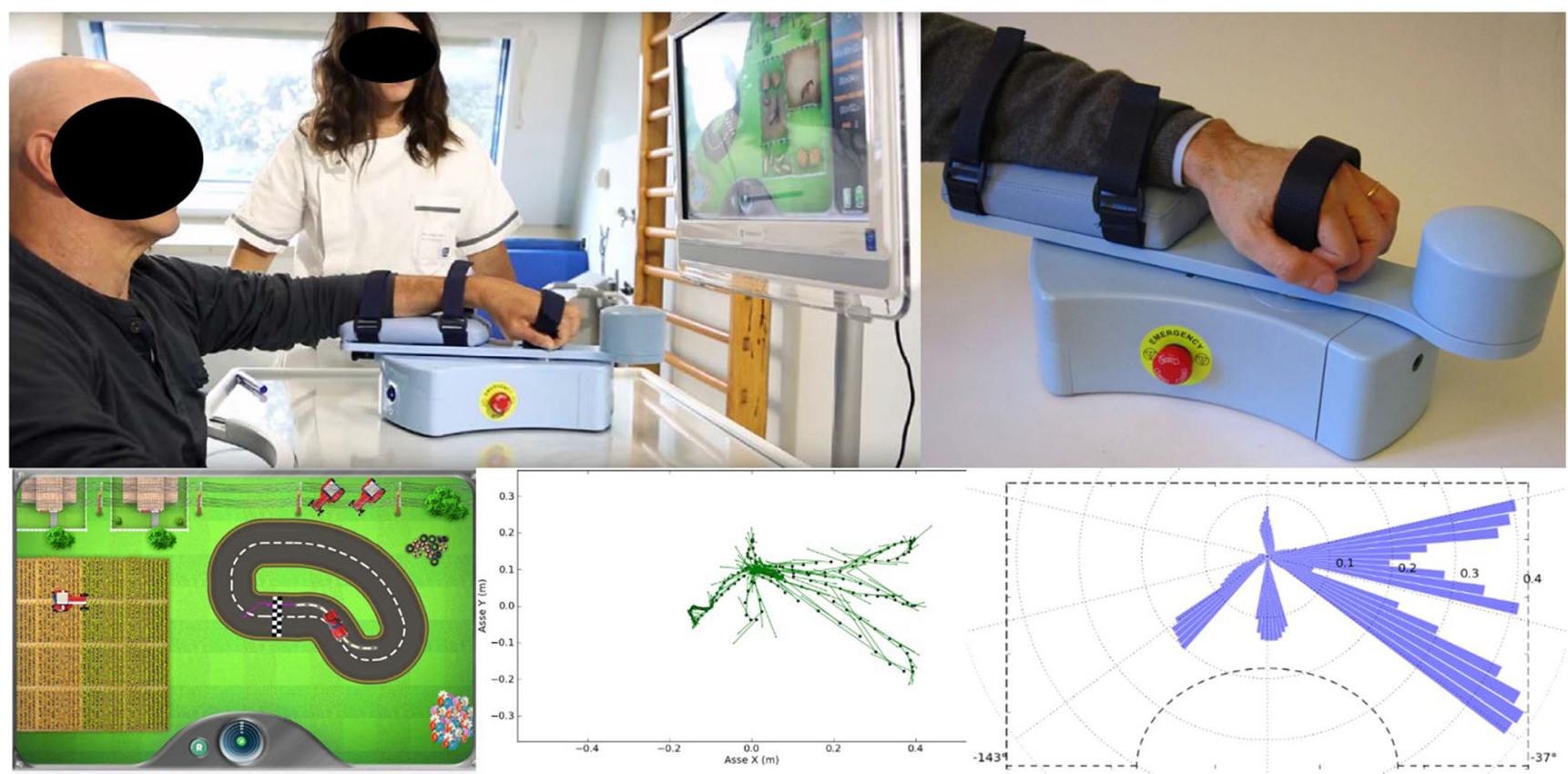

Fig. 1 MObile roboT for upper limb neuroOrtho Rehabilitation (MOTORE)

Robotic therapy was started seven days after botulinum toxin infiltration.

\section{Conventional rehabilitative treatment}

In addition, after the $30 \mathrm{~min}$ of robotic training, patients in both groups performed passive mobilization exercises on the main joints of the UL and stretching (shoulder, elbow, wrist, and fingers), scapular joint mobilization exercises, and cervical spine "pompage" exercises: the rehabilitation maneuvers were performed by the physiotherapist, expert in neurological rehabilitation, on the couch with the patient in a prone or supine position as needed. The protocol also included active movements of the UL against the force of gravity, with the final request of isometric holding of the position for at least $6 \mathrm{~s}$. According to the patient's resources, the physiotherapist, during the treatment, increased the joint range during passive mobilization and the number of repetitions, starting from a minimum of 5 repetitions up to 15 repetitions at least twice, with a rest break from 2 to $3 \mathrm{~min}$. The stretching exercises were performed statically for at least three times during the session.

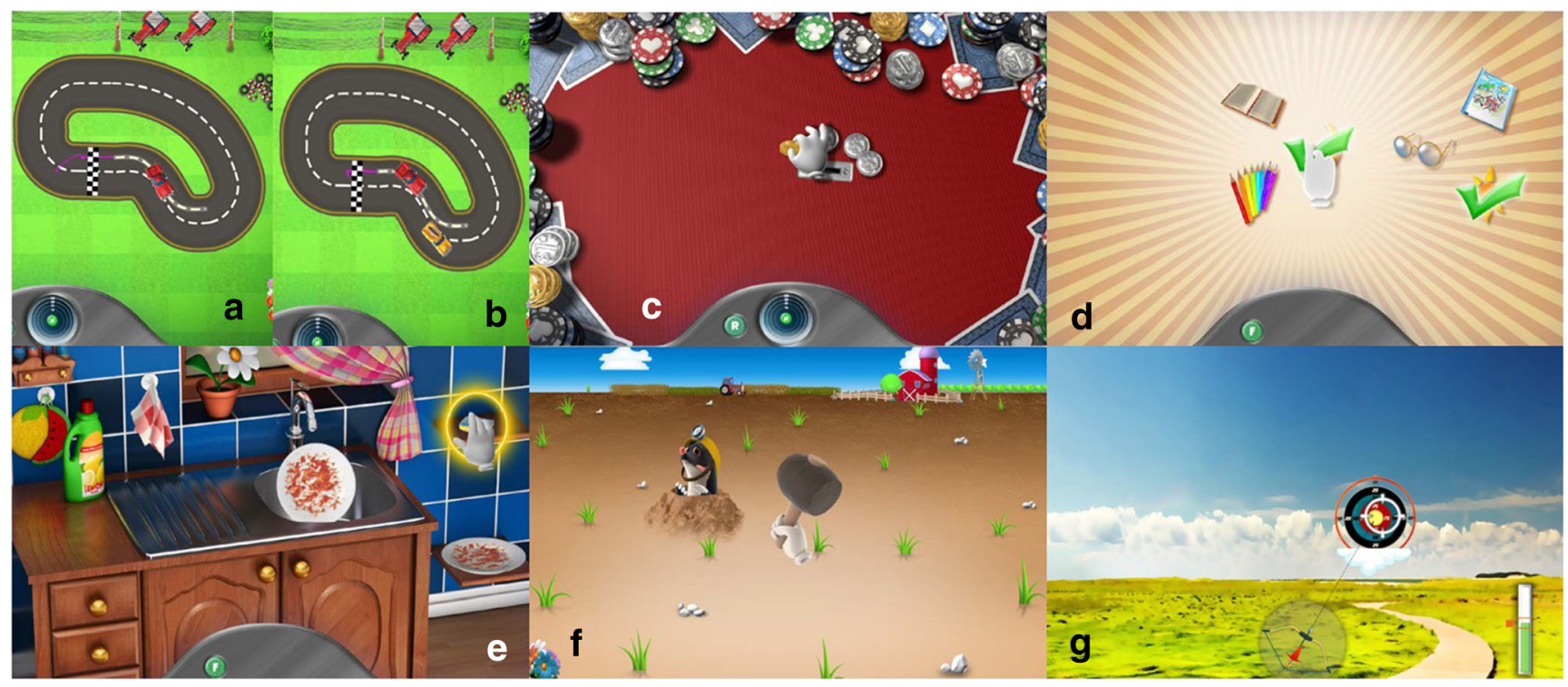

Fig. 2 Robot end-effector protocol. a Trajectories; $\mathbf{b}$ pursuit; $\mathbf{c}$ coins; $\mathbf{d}$ memory; $\mathbf{e}$ washing dishes; $\mathbf{f}$ moles; $\mathbf{g}$ archery 
Conventional treatment followed robotic treatment for organizational needs of the rehabilitation department and had the same duration as robotic therapy, to also facilitate the patient as it was an outpatient rehabilitation therapy, 3 days a week, for a total of 20 sessions

\section{Outcome measure}

Fugl-Meyer Assessment scale (FMA) The FM scale is a 226point multi-item Likert-type scale that was developed as a stroke-specific, performance-based impairment index. It is designed to assess motor functioning, balance, sensation, and joint functioning in patients with post-stroke hemiplegia. Each domain contains multiple items, each of which is scored on a 3 -point ordinal scale $(0=$ cannot perform, $1=$ performs partially, 2 = performs fully). The FMA-UL motor domain is most widely used and has the primary value of monitoring motor recovery after stroke specifically for the upper limb (maximum score 66 points) [29].

Motricity Index (MI) The muscle weakness of the paretic upper extremity was quantified using the Motricity Index. It involves grading strength based on a patient's ability to activate a muscle group, to move a limb segment through a range of motion, and to resist the force of an examiner. The scale includes three actions: pinch grasp, elbow flexion, and shoulder abduction, and these are each scored $(0-33)$ with a maximum possible score of 100 (adding one to the sum of the three actions) for the upper limb [30].

Modified Ashworth scale (MAS) The modified Ashworth scale is the most widely clinical scale used to measure muscle spasticity. It is used to assess the resistance experienced during the passive range of upper limb motion (shoulder adductors, elbow, and wrist flexors), which does not require any instrumentation and is quick to perform. The scale consists of a 5point nominal scale using subjective clinical assessments of tone ranging from 0 - "no increases in tone"- to 4- "limb rigid in flexion or extension (abduction/adduction)." An additional grade is added (1+) for the MAS to indicate resistance in the movement [31].

Numeric rating scale (NRS) Numerical rating scale (NRS) is the simplest and most commonly used scale to assess pain; the range is from 0 to 10 , with 0 being "no pain" and 10 being "the worst pain imaginable" [32].

Box and Block Test (BBT) The BBT is a quick, simple, and reliable measurement, and it is used to assess the unilateral gross manual dexterity. The BBT consists of moving the maximum number of blocks from one compartment of a box to another, one by one, within $1 \min$ [33].
Frenchay Arm Test (FAT) The FAT assesses upper extremity specific measure of activity limitation. It is an upper extremity proximal motor control and dexterity during ADL performance in patients with impairments of the upper extremity resulting from neurological conditions [34].

Barthel Index (BI) The BI was introduced by Mahoney and Barthel (1965) and later modified by Collin et al. (1988) and Shah et al. (1989). The original 10-item form of the BI comprises 10 common activities of daily living (ADL): "feeding," "bathing," "grooming," "dressing," "bowel" and "bladder control," "toilet use," "transfers (bed to chair and back)," "mobility," and "stair climbing." The items are rated as whether patients can perform the activities independently or with assistance or are totally dependent (scored 10,5 , or 0 respectively, or from 15 to 0 for transfers and mobility) [35-38].

\section{Sample size calculation}

The G*Power version 3.1.9.2 program was used to evaluate the required sample size.

We used the difference in time $(\Delta \mathrm{T})$ of both the FuglMeyer Assessment (FMA) and the Motricity Index (MI) values as an outcome for the calculation of the sample size, inserting the following values for the FMA scale mean $=5.29$, $\mathrm{SD}=5.02$ and for the MI mean $=-0.67, \mathrm{SD}=0.73[16]$; $\mathrm{a}$ significance level of 0.05 and a power level of 0.95 ; the sample size required is respectively 13 and 15 per group.

\section{Statistical analysis}

Values are expressed as median and minimum and maximum for continuous variables and proportion for categorical variables, as appropriate. Demographic and clinical data at baseline included the following parameters: age and BMI (body mass index) expressed in median and minimum and maximum, gender (female or male), hemiplegic side (left or right), and dominant side (left or right). Differences in baseline characteristics between the 2 treatment groups were analyzed by Fisher's exact test or Mann-Whitney $U$ test, as appropriate. The analysis of time difference in the two groups (robot group, RG and robot + toxin group, RTG) was performed through a Friedman analysis for repeated measures to determine if there were differences in the different evaluation times in the 2 groups. A subsequent analysis for each parameter, a pairwise comparison, was performed with a Bonferroni correction. The Mann-Whitney $U$ test was used for all the parameters studied to evaluate the time differences between the groups and the variations $(\Delta)$ between $\mathrm{T} 0$ and $\mathrm{T} 1, \mathrm{~T} 1$ and $\mathrm{T} 2$, and $\mathrm{T} 2$ and $\mathrm{T} 0$. All primary and secondary outcomes analyzed were performed according to the principle of intention-to-treat analysis. A value of $p<0.05$ was considered significant. Statistical 
analyses were carried out using the SPSS version 18 package (SPSS Inc, Chicago, IL, USA).

\section{Result}

Sixty-five $(N=65)$ patients were observed and forty-seven were enrolled respect the inclusion criteria. Specifically, data of forty-four $(N=44)$ patients were included and analyzed. We observed a dropout of 2 patients in the RG group of which 1 due to discontinuity to treatment and 1 for family reasons; on the other hand, in the RTG, we observed 1 dropout due to discontinuity in treatment. Then, the data of 21 patients in the RG and 23 patients in the RG were analyzed (Fig. 3).

Two patients in RG and one patient in RTG, respectively, did not complete the rehabilitative program: one patient in the RG was excluded because he did not constantly participate in the rehabilitation program and one for a personal problem, instead, one patient was excluded in the RTG because he did not perform all the rehabilitative sessions. No adverse events were reported during rehabilitative treatment and toxin treatment.

A descriptive analysis was performed as showed in Table 2 for age, body mass index (BMI), male/female), hemiplegic side (left or right), and dominant side (left or right). Compared to the baseline, the two groups are homogeneous and match for age with a mean age (years) of $65 \pm 10.09$ (RG, males 67\%) and
$65.7 \pm 10.5$ (RTG, males $43.5 \%$ ) for $P=0.487$, BMI $26.8 \pm 2.3$ (RG) and $26.9 \pm 1.8(\mathrm{RTG})$ for $P=0.567$.

From the analysis between groups, the results suggested how there was a statistically significant difference for $p<$ 0.05 in favor of the RTG versus RG group as reported in Table 3 , specifically $\Delta \mathrm{T} 0-\mathrm{T} 1$ and $\Delta \mathrm{T} 0$-T2 for B\&B $p=$ 0.009 and $p=0.035 ; \Delta$ T0-T1 and $\Delta$ T0-T2 for Frenchay Arm Scale with $p=0.016$ and $p=0.031 ; \Delta$ T0-T1 for MAS shoulder $p=0.016 ; \Delta$ T0-T1 and $\Delta$ T0-T2 with $p=0.010$ and $p=0.005$ for MAS elbow; and $\Delta \mathrm{T} 0$-T1 and $\Delta$ T0-T2 with $p$ $=0.001$ and $p=0.013$ for MAS wrist.

From the analysis within group, the results showed an improvement over time compared for all parameters in the RTG; instead, in the $\mathrm{RG}$, the results underlined a constant trend for MAS and NRS without a statistically significant difference over time (Table 4).

\section{Discussion}

Considering the scope of our research, the results were encouraging. The data reported confirm the validity of robotic-assisted therapy for the rehabilitation of the UL in the chronic poststroke patient: both groups report a statistically significant improvement after treatment with the EE system for the FMA scale. The enrolled patients also demonstrated good compliance to the rehabilitation treatment (dropout $<20 \%$ ), reporting no

Fig. 3 Flowchart

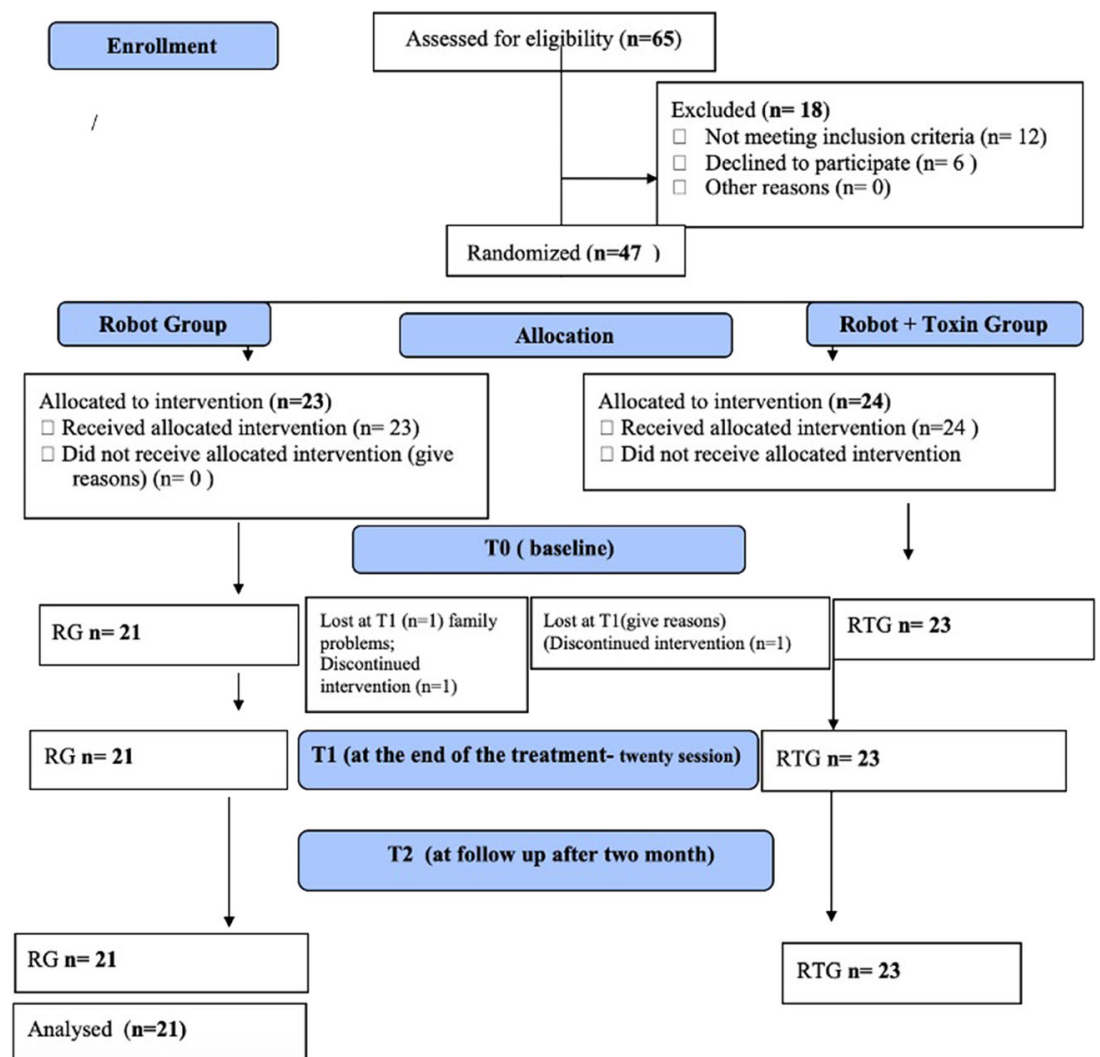


Table 2 Clinical parameters

\begin{tabular}{|c|c|c|c|}
\hline Clinical parameters & Robot group (21) & Robot + toxin group (23) & $P$ value \\
\hline Age (years-mean standard deviation)) & $65 \pm 10.09$ & $65.7 \pm 10.5$ & 0.487 \\
\hline BMI (mean standard deviation) & $26.8 \pm 2.3$ & $26.9 \pm 1.8$ & 0.567 \\
\hline Gender $(\%)$ & $67 \mathrm{M} ; 33 \mathrm{~F}$ & $43.5 \mathrm{M} ; 56.5 \mathrm{~F}$ & 0.122 \\
\hline Hemiplegic side (\%) & 57 left; 43 right & 56.5 left; 43.5 right & 0.309 \\
\hline Dominant side $(\%)$ & 95 right; 5 left & 100 right; 0 left & - \\
\hline Type of stroke (\%) & $\begin{array}{l}\text { 14.3\% TACS*; } 66.7 \% \text { PACS*; } \\
\text { 19.0 hemorrhagic stroke }\end{array}$ & $\begin{array}{l}\text { 30.4\% TACS*; } 56.5 \% \text { PACS*; } \\
13.1 \text { hemorrhagic stroke }\end{array}$ & 0.724 \\
\hline FMA (median, range) & $37(16-58)$ & $34(23-54)$ & 0.759 \\
\hline MAS shoulder (median, range) & $1(1-4)$ & $1(1-4)$ & 0.159 \\
\hline MAS elbow (median, range) & $1(1-4)$ & $2(1-4)$ & 0.163 \\
\hline MAS wrist (median, range) & $1(1-4)$ & $2(1-4)$ & 0.184 \\
\hline $\begin{array}{l}\text { Time from stroke } \\
\text { (months-mean standard deviation) }\end{array}$ & $8.5 \pm 2.6$ & & \\
\hline
\end{tabular}

$B M I$, body mass index; mean and standard deviation; TACS, total anterior circulation stroke; PACS, partial anterior circulation stroke

*Bamford classification

adverse effects in the group subjected to infiltration with BoTN. Also, no side effects have been described also for robotic rehabilitation as well as for infiltration with BoTN.

The EE system allowed the integration of cognitive stimuli with motor stimuli through feedback stimulus (as visual and auditory cues), biofeedback, VR, and exergame augmented feedback during training exercises. Thus, during exercise robot training, the bottom-up motor rehabilitation component (motor intensity and task oriented) and the top-down (attention, visuo-spatial ability) motor cognitive rehabilitation component are integrated with robots [39]. Robotic treatment should be considered a rehabilitation tool useful to generate a more complex, controlled multisensory stimulation of the patient and useful to modify the plasticity of neural connections through the experience of movement [6].

Our results, underline how, in the experimental group (RTG) where the infiltration with BoTN was carried out before robot training, parallel to the reduction of spasticity, a statistically significant difference was observed between the two groups not only for the MAS (shoulder, elbow, and wrist) at $\mathrm{T} 1$ and for the MAS wrist also at $\mathrm{T} 2$ but also compared to manual dexterity for the $\mathrm{B} \& \mathrm{~B}$ scale at $\mathrm{T} 1$ and $\mathrm{T} 2$ and the Frenchay Arm Scale, always at both T1 and T2.

Furthermore, the treatment with BoTN would have a different action also in the remodeling of the plasticity of the recovery of the UL movement, in patients with chronic stroke [40]: different effects of BoNT lowered spasticity on sensorimotor networks for mild or severe weakness in hand weakness.

Considering the study by Gandolfi et al., the results suggested an increase in muscle strength on UL in the robotassisted UL group, an improvement in shoulder range of motion in particular abduction and external rotation, and an improvement in elbow flexion. In this study, having both the experimental and control groups, treated with BoNT, no differences in the MAS and FMA scales were reported. Compared to our protocol, the authors proposed a robotassisted treatment for UL of two sessions/week lasting $45 \mathrm{~min}$ for 10 sessions in total, while our protocol envisaged three sessions per week for a total of 20 sessions. In addition, in our integrated rehabilitative approach, after the 30-min robotic session, patients in both groups performed passive mobilization exercises on the main joints of the UL and stretching [17].

We cannot forget that in chronic stroke patients, spasticity and the reduction of UL function represent one of the main interventions of the rehabilitation requests. Furthermore, in the first year after a stroke, up to $38 \%$ of patients may report spasticity of the UL (range from 7 to $38 \%$ ), and the precocity of the targeted rehabilitation intervention can avoid the onset of viscoelastic changes in the muscle and soft tissue, which would affect the joint recovery of the UL [41-44].

The robot training in the recovery of UL respects the characteristics of being task specific, repetitive, and motivating for the patients, who often have depressive disorders and poor alliance to rehabilitation treatment [45-47].

Other authors, such as Pennati et al., adopt a rehabilitation protocol of 10 sessions with robot training lasting $60 \mathrm{~min}$, two or three times a week: the data showed that in some chronic stroke patients with focal UL spasticity, botulinum toxin does not add further improvements if performed together with robotic training. These results, although very interesting, must be interpreted in the light of the study design as a pilot with a small sample size [16]. However, the starting hypothesis is supported by the literature and embraces both the importance of how the treatment of UL spasticity should always consider both the neurogenic component and the peripheral component of spasticity and of how robotic training does not increase hypertonia but reduce the spasticity of the antagonist muscles, through a mechanism of reciprocal inhibition [48-51]. 
Table 3 Statistical analysis between groups

\begin{tabular}{|c|c|c|c|}
\hline & Robot group & Robot + toxin group & $p$ value \\
\hline$\Delta \mathrm{T} 0-\mathrm{T} 1 \mathrm{FMA}$ & $10(-1-28)$ & $7(1-27)$ & 0.689 \\
\hline$\Delta \mathrm{T} 1-\mathrm{T} 2 \mathrm{FMA}$ & $2(0-20)$ & $4(-2-13)$ & 0.558 \\
\hline$\Delta \mathrm{T} 0-\mathrm{T} 2 \mathrm{FMA}$ & $12(2-30)$ & $15(3-38)$ & 0.972 \\
\hline$\Delta \mathrm{T} 0-\mathrm{T} 1 \mathrm{MI}$ UL & $16(0-50)$ & $15(3-41)$ & 0.317 \\
\hline$\Delta \mathrm{T} 1-\mathrm{T} 2 \mathrm{MI} \mathrm{UL}$ & $0(0-24)$ & $2(0-10)$ & 0.210 \\
\hline$\Delta \mathrm{T} 0-\mathrm{T} 2 \mathrm{MI} \mathrm{UL}$ & $18(0-50)$ & $20(7-43)$ & 0.503 \\
\hline$\Delta \mathrm{T} 0-\mathrm{T} 1 \mathrm{~B} \& \mathrm{~B}$ & $5(-11-49)$ & $12(4-31)$ & 0.009 \\
\hline$\Delta \mathrm{T} 1-\mathrm{T} 2 \mathrm{~B} \& \mathrm{~B}$ & $0(-2-22)$ & $2(0-12)$ & 0.077 \\
\hline$\Delta \mathrm{T} 0-\mathrm{T} 2 \mathrm{~B} \& \mathrm{~B}$ & $9(0-49)$ & $16(7-36)$ & 0.035 \\
\hline$\Delta \mathrm{T} 0$-T1 Frenchay Arm & $1(0-5)$ & $2(0-4)$ & 0.016 \\
\hline$\Delta \mathrm{T} 1-\mathrm{T} 2$ Frenchay Arm & $0(0-2)$ & $0(0-1)$ & 0.249 \\
\hline$\Delta \mathrm{T} 0$-T2 Frenchay Arm & $1(0-5)$ & $2(0-5)$ & 0.031 \\
\hline$\Delta \mathrm{T} 0$-T1 MAS shoulder & $0(-3-1)$ & $-1(-3-1)$ & 0.016 \\
\hline$\Delta \mathrm{T} 1-\mathrm{T} 2 \mathrm{MAS}$ shoulder & $0(-1-2)$ & $0(-1-1)$ & 0.576 \\
\hline$\Delta \mathrm{T} 0$-T2 MAS shoulder & $0(-3-2)$ & $-1(-3-1)$ & 0.054 \\
\hline$\Delta \mathrm{T} 0-\mathrm{T} 1 \mathrm{MAS}$ elbow & $0(-2-2)$ & $-1(-3-0)$ & 0.010 \\
\hline$\Delta \mathrm{T} 1-\mathrm{T} 2 \mathrm{MAS}$ elbow & $0(-3-2)$ & $0(-1-0)$ & 0.282 \\
\hline$\Delta \mathrm{T} 0-\mathrm{T} 2 \mathrm{MAS}$ elbow & $0(-2-1)$ & $-1(-4-0)$ & 0.005 \\
\hline$\Delta \mathrm{T} 0$-T1 MAS wrist & $0(-4-2)$ & $-1(-4-1)$ & 0.001 \\
\hline$\Delta \mathrm{T} 1-\mathrm{T} 2 \mathrm{MAS}$ wrist & $0(-2-2)$ & $0(-1-0)$ & 0.176 \\
\hline$\Delta \mathrm{T} 0$-T2 MAS wrist & $0(-4-2)$ & $-1(-4-0)$ & 0.013 \\
\hline$\Delta \mathrm{T} 0-\mathrm{T} 1 \mathrm{NRS}$ & $0(-6-5)$ & $-2(-8-1)$ & 0.258 \\
\hline$\Delta \mathrm{T} 1-\mathrm{T} 2 \mathrm{NRS}$ & $0(-3-2)$ & $0(-1-0)$ & 0.366 \\
\hline$\Delta \mathrm{T} 0-\mathrm{T} 2 \mathrm{NRS}$ & $0(-6-2)$ & $-2(-8-1)$ & 0.188 \\
\hline$\Delta \mathrm{T} 0-\mathrm{T} 1 \mathrm{BI}$ & $10(0-65)$ & $16(0-53)$ & 0.715 \\
\hline$\Delta \mathrm{T} 1-\mathrm{T} 2 \mathrm{BI}$ & $0(-15-45)$ & $2(0-10)$ & 0.668 \\
\hline$\Delta \mathrm{T} 0-\mathrm{T} 2 \mathrm{BI}$ & $25(-10-80)$ & $19(4-57)$ & 0.525 \\
\hline
\end{tabular}

Legends: $\Delta=$ difference; FMA, Fugl-Meyer Assessment scale; MI, Motricity Index; MAS, modified Ashworth scale; NRS, numeric rating scale; $B B T$, Box and Block Test; FAT, Frenchay Arm Test; $B I$, Barthel Index; baseline (T0), T1 (at the end of the treatment), and T2 (after 3 months of follow-up)

In a recent multi-center study by Aprile et al., robot training for UL was performed in the rehabilitation of stroke patients with a daily frequency ( 5 days a week) for $45 \mathrm{~min}$, for a total of 30 sessions. The authors reported that there are no statistically significant differences in functional recovery of UL in patients with subacute stroke, the outcomes being equivalent with respect to conventional rehabilitative therapy. Instead, it was highlighted that, in the group of patients who carried out the robotic training, the subjects reported an increase in strength on the UL, probably related to the high number of repetitions and to a more intense training [27]. Also, in patients with long-term upper limb deficits after stroke, other authors suggested a similar trend with a better result for 12-week robot-assisted therapy group with respect to usual care for FM score but the differences were not significant [52].
From the analysis within group, the results showed an improvement over time compared for all parameters in the RTG; instead, in the RG, the results underlined a constant trend for MAS and NRS without a statistically significant difference over time (Table 4). Yelnik et al. [53], in their randomized, double-blind, placebo-controlled, two-parallel-group study investigated the beneficial effect of injection of botulinum toxin A (single-dose botulinum toxin-500 Speywood units) into the muscle subscapularis for shoulder pain in stroke patients with spastic hemiplegia. Pain was assessed using a 10-point verbal scale. Upper limb spasticity was assessed using the MAS for the medial rotators of the shoulder and for the flexors of the elbow, wrist, and fingers. Assessments were performed at baseline and at weeks 1,2, and 4. A clinically significant improvement in the passive lateral rotation was observed, resulting from a decrease in local spasticity. External rotation is greatly improved, more so than abduction, which is not surprising because the subscapularis muscle is a strong internal rotator with little impact on abduction. In conjunction with the improvement in shoulder pain and mobility, spasticity of the upper limb muscles appeared to be reduced. The authors conclude that subscapular injection of botulinum toxin A appears to be useful in the management of shoulder pain in spastic hemiplegic patients. The reduction of pain by subscapular injection of botulinum toxin A, with a concomitant improvement in the range of motion of the shoulder observed in the present study, therefore, in line with our results, confirms the role of spasticity in post-stroke shoulder pain.

Limits The positive effect of this therapy with respect to neuroplasticity is hypothesized but not studied in this paper. The only clinical analysis (or with a screening tool such as the Mini Mental) does not allow a more reliable verification of the percentage of subjects who cannot access this treatment due to neuropsychological limitations. In this study, muscle recruitment with electromyography (EMG) was not assessed. The absence of instrumental evaluation (for example h-reflex, dynamometer, gait analysis, or correlation between the variation of spasticity and muscle strength) of the observed phenomena certainly represents a limitation. The clinical judgment alone was used to investigate the presence of neglect. The assessments at T0, T1, and T2 and the related statistical results between and within the groups, using the "MOTORE" test and related data (force, acceleration, speed), were not included in the manuscript because we focused on the functional recovery of the patients with chronic stroke. The lack of analysis of the level of aphasia represents a limitation.

\section{Conclusion}

The spasticity of UL together with functional reduction and painful, in chronic stroke patients, is the primary reason for the 
Table 4 Statistical analysis within group

\begin{tabular}{|c|c|c|c|c|c|c|c|c|}
\hline & \multirow[t]{2}{*}{ Group } & \multicolumn{4}{|c|}{ Evaluation times } & \multicolumn{3}{|l|}{ Post hoc } \\
\hline & & T0 & $\mathrm{T} 1$ & $\mathrm{~T} 2$ & $p$ value & $p$ T0-T1 & $p$ T1-T2 & $p$ T0-T2 \\
\hline FMA & Robot group & $37(16-58)$ & $46(20-65)$ & $55(20-65)$ & $<0.001$ & 0.001 & 0.160 & $<0.001$ \\
\hline MI UL & Robot group & $50(28-76)$ & $66(28-100)$ & $66(28-100)$ & $<0.001$ & 0.002 & 0.370 & $<0.001$ \\
\hline $\mathrm{B} \& \mathrm{~B}$ & Robot group & $11(0-34)$ & $23(0-53)$ & $27(0-53)$ & $<0.001$ & 0.005 & 0.570 & $<0.001$ \\
\hline Frenchay arm & Robot group & $2(0-5)$ & $4(0-7)$ & $4(0-7)$ & $<0.001$ & 0.050 & 0.950 & 0.002 \\
\hline MAS shoulder & Robot group & $1(1-4)$ & $1(0-2)$ & $0(0-3)$ & 0.900 & - & - & - \\
\hline MAS elbow & Robot group & $1(1-4)$ & $1(0-3)$ & $1(0-3)$ & 0.050 & - & - & - \\
\hline MAS wrist & Robot group & $1(1-4)$ & $1(0-4)$ & $0(0-4)$ & 0.060 & - & - & - \\
\hline NRS & Robot group & $1(0-10)$ & $0(0-10)$ & $0(0-10)$ & 0.060 & - & - & - \\
\hline BI & Robot group & $65(20-90)$ & $80(30-100)$ & $90(55-100)$ & $<0.001$ & 0.002 & 1 & $<0.001$ \\
\hline FMA & Robot + toxin group & $34(23-54)$ & $49(31-65)$ & $52(34-72)$ & $<0.001$ & $<0.001$ & 0.060 & $<0.001$ \\
\hline MI UL & Robot + toxin group & $50(28-72)$ & $67(42-100)$ & $68(44-100)$ & $<0.001$ & 0.001 & 0.001 & $<0.001$ \\
\hline $\mathrm{B} \& \mathrm{~B}$ & Robot + toxin group & $4(0-33)$ & $23(6-53)$ & $26(12-58)$ & $<0.001$ & 0.002 & 0.004 & $<0.001$ \\
\hline Frenchay arm & Robot + toxin group & $2(0-5)$ & $3(2-5)$ & $4(3-5)$ & $<0.001$ & 0.001 & 0.314 & $<0.001$ \\
\hline MAS shoulder & Robot + toxin group & $1(1-4)$ & $0(0-1)$ & $0(0-1)$ & $<0.001$ & 0.012 & 1 & 0.045 \\
\hline MAS elbow & Robot + toxin group & $2(1-4)$ & $1(0-2)$ & $0(0-1)$ & $<0.001$ & 0.015 & 1 & 0.001 \\
\hline MAS wrist & Robot + toxin group & $2(1-4)$ & $0(0-2)$ & $0(0-1)$ & $<0.001$ & $<0.001$ & 1 & $<0.001$ \\
\hline NRS & Robot + toxin group & $2(0-10)$ & $1(0-4)$ & $1(0-3)$ & 0.001 & 0.081 & 1 & 0.012 \\
\hline BI & Robot + toxin group & $67(25-92)$ & $82(58-100)$ & $82(60-100)$ & 0.007 & - & - & - \\
\hline
\end{tabular}

Legends: FMA, Fugl-Meyer Assessment scale; MI, Motricity Index; MAS, modified Ashworth scale; NRS, numeric rating scale; BBT, Box and Block Test; FAT, Frenchay Arm Test; BI, Barthel Index (BI); baseline (T0), T1 (at the end of the treatment), and T2 (after 3 months of follow-up)

request for rehabilitation intervention. Our results suggest, in line with the literature, a good efficacy in the reduction of spasticity and in the improvement of the function of the UL, with the reduction of UL pain, adopting a rehabilitation protocol integrated with BoTN, robot-assisted training, and traditional physiotherapy.

Acknowledgements We want to thank the occupational therapist Simona Di Federico, who made a great contribution to the success of the study, of U.O.C. Physical Medicine and Rehabilitation - Hospital of Popoli, Pescara, Italy.

Funding Open access funding provided by Università degli Studi di Roma La Sapienza within the CRUI-CARE Agreement.

\section{Declarations}

Conflict of interest The authors declare no competing interests.

Ethical approval The research was approved by the Local Hospital Committee of Popoli and was approved by the Department of Oral and Biotechnological MedicalSciences of the G. D'Annunzio University of Chieti (Italy) (315/20).

Open Access This article is licensed under a Creative Commons Attribution 4.0 International License, which permits use, sharing, adaptation, distribution and reproduction in any medium or format, as long as you give appropriate credit to the original author(s) and the source, provide a link to the Creative Commons licence, and indicate if changes were made. The images or other third party material in this article are included in the article's Creative Commons licence, unless indicated otherwise in a credit line to the material. If material is not included in the article's Creative Commons licence and your intended use is not permitted by statutory regulation or exceeds the permitted use, you will need to obtain permission directly from the copyright holder. To view a copy of this licence, visit http://creativecommons.org/licenses/by/4.0/.

\section{References}

1. Kwakkel G, Kollen BJ, van der Grond J, Prevo AJ (2003) Probability of regaining dexterity in the flaccid upper limb: impact of severity of paresis and time since onset in acute stroke. Stroke. 34(9):2181-2186

2. Broeks JG, Lankhorst GJ, Rumping K, Prevo AJ (1999) The longterm outcome of arm function after stroke: results of a follow-up study. Disabil Rehabil 21(8):357-364

3. Hebert D, Lindsay MP, McIntyre A, Kirton A, Rumney PG, Bagg S, Bayley M, Dowlatshahi D, Dukelow S, Garnhum M, Glasser E, Halabi ML, Kang E, MacKay-Lyons M, Martino R, Rochette A, Rowe S, Salbach N, Semenko B, Stack B, Swinton L, Weber V, Mayer M, Verrilli S, DeVeber G, Andersen J, Barlow K, Cassidy C, Dilenge ME, Fehlings D, Hung R, Iruthayarajah J, Lenz L, Majnemer A, Purtzki J, Rafay M, Sonnenberg LK, Townley A, Janzen S, Foley N, Teasell R (2016) Canadian stroke best practice recommendations: stroke rehabilitation practice guidelines, update 2015. Int J Stroke 11(4):459-484

4. Oujamaa L, Relave I, Froger J, Mottet D, Pelissier JY (2009) Rehabilitation of arm function after stroke. Literature review. Ann Phys Rehabil Med 52(3):269-293 
5. Raghavan P (2015) Upper limb motor impairment after stroke. Phys Med Rehabil Clin N Am 26(4):599-610

6. Molteni F, Gasperini G, Cannaviello G, Guanziroli E (2018) Exoskeleton and end-effector robots for upper and lower limbs rehabilitation: narrative review. PM R 10(9 Suppl 2):S174-S188

7. Valk TA, Mouton LJ, Otten E, Bongers RM (2019) Synergies reciprocally relate end-effector and joint-angles in rhythmic pointing movements. Sci Rep 9(1):17378 Published 2019 Nov 22

8. Makinde OA, Mpofu K, Vrabic R, Ramatsetse BI (2017) A bioinspired approach for the design of a multifunctional robotic endeffector customized for automated maintenance of a reconfigurable vibrating screen. Robotics Biomim 4(1):4

9. Liang K, Xing Y, Li J, Wang S, Li A, Li J (2018) Motion control skill assessment based on kinematic analysis of robotic end-effector movements. Int J Med Robot 14(1). https://doi.org/10.1002/rcs. 1845

10. Bonita R, Beaglehole R (1988) Recovery of motor function after stroke. Stroke 19(12):1497-1500

11. Pollock A, Farmer SE, Brady MC, Langhorne P, Mead GE, Mehrholz J, van Wijck F (2014) Interventions for improving upper limb function after stroke. Cochrane Database Syst Rev : CD010820. https://doi.org/10.1002/14651858.CD010820.pub2

12. Dehem S, Gilliaux M, Stoquart G, Detrembleur C, Jacquemin G, Palumbo S, Frederick A, Lejeune T (2019) Effectiveness of upperlimb robotic-assisted therapy in the early rehabilitation phase after stroke: a single-blind, randomised, controlled trial. Ann Phys Rehabil Med 62(5):313-320

13. Liu Y, Song Q, Li C, Guan X, Ji L (2020) Quantitative assessment of motor function for patients with a stroke by an end-effector upper limb rehabilitation robot. Biomed Res Int :5425741. https://doi.org/ 10.1155/2020/5425741

14. Lee SH, Park G, Cho DY, Kim HY, Lee JY, Kim S, Park SB, Shin JH (2020) Comparisons between end-effector and exoskeleton rehabilitation robots regarding upper extremity function among chronic stroke patients with moderate-to-severe upper limb impairment. Sci Rep 10(1):1806

15. Mazzoleni S, Battini E, Crecchi R, Dario P, Posteraro F (2018) Upper limb robot-assisted therapy in subacute and chronic stroke patients using an innovative end-effector haptic device: a pilot study. NeuroRehabilitation. 42(1):43-52

16. Pennati GV, Da Re C, Messineo I, Bonaiuti D (2015) How could robotic training and botolinum toxin be combined in chronic post stroke upper limb spasticity? A pilot study. Eur J Phys Rehabil Med 51(4):381-387

17. Gandolfi M, Valè N, Dimitrova EK, Mazzoleni S, Battini E, Filippetti M, Picelli A, Santamato A, Gravina M, Saltuari L, Smania N (2019) Effectiveness of robot-assisted upper limb training on spasticity, function and muscle activity in chronic stroke patients treated with botulinum toxin: a randomized singleblinded controlled trial. Front Neurol 10:41

18. Dong Y, Wu T, Hu X, Wang T (2017) Efficacy and safety of botulinum toxin type A for upper limb spasticity after stroke or traumatic brain injury: a systematic review with meta-analysis and trial sequential analysis. Eur J Phys Rehabil Med 53(2):256-267

19. Sun LC, Chen R, Fu C, Chen Y, Wu Q, Chen R, Lin X, Luo S (2019) Efficacy and safety of botulinum toxin type A for limb spasticity after stroke: a meta-analysis of randomized controlled trials. Biomed Res Int 2019:8329306

20. Andringa A, van de Port I, van Wegen E, Ket J, Meskers C, Kwakkel G (2019) Effectiveness of botulinum toxin treatment for upper limb spasticity poststroke over different ICF domains: a systematic review and meta-analysis. Arch Phys Med Rehabil 100(9): $1703-1725$

21. Serra MC (2018) The importance of assessing nutritional status to ensure optimal recovery during the chronic phase of stroke. Stroke Res Treat 2018:1297846
22. Li H, Jia J, Yang Z (2016) Mini-Mental State Examination in elderly Chinese: a population-based normative study. J Alzheimers Dis 53(2):487-496

23. Carpinelli Mazzi M, Iavarone A, Russo G, Musella C, Milan G, D’Anna F, Garofalo E, Chieffi S, Sannino M, Illario M, De Luca V, Postiglione A, Abete $\mathrm{P}$, with the support of the Working group (2020) Mini-Mental State Examination: new normative values on subjects in Southern Italy. Aging Clin Exp Res 32(4):699-702

24. Frisoni GB, Rozzini R, Bianchetti A, Trabucchi M (1993) Principal lifetime occupation and MMSE score in elderly persons. J Gerontol 48(6):S310-S314

25. von Elm E, Altman DG, Egger M, Pocock SJ, Gøtzsche PC, Vandenbroucke JP, STROBE Initiative (2007) The Strengthening the Reporting of Observational Studies in Epidemiology (STROBE) statement: guidelines for reporting observational studies. Ann Intern Med 147(8):573-577

26. Kara M, Kaymak B, Ulașli AM, Tok F, Öztürk GT, Chang KV, Hsiao MY, Hung CY, Yağiz On A, Özçakar L (2018) Sonographic guide for botulinum toxin injections of the upper limb: EUROMUSCULUS/USPRM spasticity approach. Eur J Phys Rehabil Med 54(3):469-485

27. Aprile I, Germanotta M, Cruciani A, Loreti S, Pecchioli C, Cecchi F, Montesano A, Galeri S, Diverio M, Falsini C, Speranza G, Langone E, Papadopoulou D, Padua L, Carrozza MC, FDG Robotic Rehabilitation Group (2020) Upper limb robotic rehabilitation after stroke: a multicenter, randomized clinical trial. J Neurol Phys Ther 44(1):3-14

28. Germanotta M, Cruciani A, Pecchioli C, Loreti S, Spedicato A, Meotti M, Mosca R, Speranza G, Cecchi F, Giannarelli G, Padua L, Aprile I (2018) Reliability, validity and discriminant ability of the instrumental indices provided by a novel planar robotic device for upper limb rehabilitation. J Neuroeng Rehabil 15(1):39

29. Gladstone DJ, Danells CJ, Black SE (2002) The Fugl-Meyer Assessment of motor recovery after stroke: a critical review of its measurement properties. Neurorehabil Neural Repair 16:232-240

30. Bohannon RW (1999) Motricity index scores are valid indicators of paretic upper extremity strength following stroke. J Phys Ther Sci 11(2):59-61

31. Gregson JM, Leathley MJ, Moore AP, Smith TL, Sharma AK, Watkins CL (2000) Reliability of measurements of muscle tone and muscle power in stroke patients. Age Ageing 29(3):223-228

32. Price DD, Bush FM, Long S, Harkins SW (1994) A comparison of pain measurement characteristics of mechanical visual analogue and simple numerical rating scales. Pain. 56:217-226

33. Mathiowetz V, Volland G, Kashman N, Weber K (1985) Adult norms for the Box and Block Test of manual dexterity. Am J Occup Ther 39(6):386-391

34. Wade DT, Langton-Hewer R, Wood VA, Skilbeck CE, Ismail HM (1983) The hemiplegic arm after stroke: measurement and recovery. J Neurol Neurosurg Psychiatry 46(6):521-524

35. Quinn TJ, Langhorne P, Stott DJ (2011) Barthel index for stroke trials: development, properties, and application. Stroke 42(4):11461151

36. Shah S, Vanclay F, Cooper B (1989) Improving the sensitivity of the Barthel Index for stroke rehabilitation. J Clin Epidemiol 42(8): 703-709

37. Damiani C, Mangone M, Paoloni M, Goffredo M, Franceschini M, Servidio M, Pournajaf S, Santilli V, Agostini F, Bernetti A (2020) Trade-offs with rehabilitation effectiveness (REs) and efficiency (REy) in a sample of Italian disabled persons in a in post-acuity rehabilitation unit. Ann Ig 32(4):327-335

38. Bernetti A, Mangone M, Alviti F, Paolucci T, Attanasi C, Murgia M, Di Sante L, Agostini F, Vitale M, Paoloni M (2020) Spa therapy and rehabilitation of musculoskeletal pathologies: a proposal for best practice in Italy. Int J Biometeorol 64(6):905-914 
39. Poli P, Morone G, Rosati G, Masiero S (2013) Robotic technologies and rehabilitation: new tools for stroke patients' therapy. Biomed Res Int 153872. https://doi.org/10.1155/2013/153872

40. Veverka T, Hluštík P, Hok P, Otruba P, Tüdös Z, Zapletalová J, Krobot A, Kaňovský P (2014) Cortical activity modulation by botulinum toxin type $\mathrm{A}$ in patients with post-stroke arm spasticity: real and imagined hand movement. J Neurol Sci 346(1-2):276-283

41. Harris JE, Eng JJ (2010) Strength training improves upper-limb function in individuals with stroke: a meta-analysis. Stroke. 41(1): $136-140$

42. Opheim A, Danielsson A, Alt Murphy M, Persson HC, Sunnerhagen KS (2014) Upper-limb spasticity during the first year after stroke: stroke arm longitudinal study at the University of Gothenburg. Am J Phys Med Rehabil 93(10):884-896

43. Smania N, Picelli A, Munari D, Geroin C, Ianes P, Waldner A, Gandolfi M (2010) Rehabilitation procedures in the management of spasticity. Eur J Phys Rehabil Med 46(3):423-438

44. Gracies JM (2005) Pathophysiology of spastic paresis. I: Paresis and soft tissue changes. Muscle Nerve 31(5):535-551

45. Masiero S, Celia A, Rosati G, Armani M (2007) Robotic-assisted rehabilitation of the upper limb after acute stroke. Arch Phys Med Rehabil 88(2):142-149

46. Lin FH, Yih DN, Shih FM, Chu CM (2019) Effect of social support and health education on depression scale scores of chronic stroke patients. Medicine (Baltimore) 98(44):e17667

47. Knepley KD, Mao JZ, Wieczorek P, Okoye FO, Jain AP, Harel NY (2020) Impact of telerehabilitation for stroke-related deficits. Telemed J E Health 27(3):239-246. https://doi.org/10.1089/tmj. 2020.0019
48. Posteraro F, Mazzoleni S, Aliboni S, Cesqui B, Battaglia A, Carrozza MC, Dario P, Micera S (2010) Upper limb spasticity reduction following active training: a robot-mediated study in patients with chronic hemiparesis. J Rehabil Med 42(3):279-281

49. Fazekas G, Horvath M, Troznai T, Toth A (2007) Robot-mediated upper limb physiotherapy for patients with spastic hemiparesis: a preliminary study. J Rehabil Med 39(7):580-582

50. Picelli A, Santamato A, Chemello E, Cinone N, Cisari C, Gandolfi M, Ranieri M, Smania N, Baricich A (2019) Adjuvant treatments associated with botulinum toxin injection for managing spasticity: an overview of the literature. Ann Phys Rehabil Med 62(4):291296

51. Li S (2017) Spasticity, motor recovery, and neural plasticity after stroke. Front Neurol 8:120

52. Lo AC, Guarino PD, Richards LG, Haselkorn JK, Wittenberg GF, Federman DG, Ringer RJ, Wagner TH, Krebs HI, Volpe BT, Bever CT Jr, Bravata DM, Duncan PW, Corn BH, Maffucci AD, Nadeau SE, Conroy SS, Powell JM, Huang GD, Peduzzi P (2010) Robotassisted therapy for long-term upper-limb impairment after stroke. N Engl J Med 362(19):1772-1783

53. Yelnik AP, Colle FM, Bonan IV, Vicaut E (2007) Treatment of shoulder pain in spastic hemiplegia by reducing spasticity of the subscapular muscle: a randomised, double blind, placebo controlled study of botulinum toxin A. J Neurol Neurosurg Psychiatry 78(8): $845-848$

Publisher's note Springer Nature remains neutral with regard to jurisdictional claims in published maps and institutional affiliations. 